\title{
Time-Resolved Dual Frequency Comb Phase Spectroscopy of Laser-Induced Plasmas
}

\author{
Reagan R. D. Weeks ${ }^{1}$, Yu Zhang ${ }^{1,2}$, Caroline Lecaplain ${ }^{1}$, Jeremy Yeak ${ }^{3}$, Sivanandan S. Harilal ${ }^{4}$, \\ Mark C. Phillips ${ }^{4}$, R. Jason Jones ${ }^{1}$ \\ 1. College of Optical Sciences, University of Arizona, 1630 E. University Blvd, Tucson, AZ 85721, USA \\ 2. Department of Physics, University of Arizona, 1118 E. Fourth Street, Tucson, AZ 85721, USA \\ 3. Opticslah, Albuquerque, NM, USA \\ 4. Pacific Northwest National Laboratory, Richland, WA 99352, USA \\ Email: rjjones@optics.arizona.edu
}

\begin{abstract}
We present the first results using time-resolved dual-comb phase spectroscopy in a laserinduced plasma. It can allow for simultaneous plasma characterization as well as multi-species detection and plasma characterization.
\end{abstract}

OCIS codes: (300.6500) Spectroscopy, time-resolved, (280.5395) Plasma diagnostics, (320.7150) Ultrafast spectroscopy

Laser-induced plasmas provide a powerful tool for analysis of solid materials. It has been widely used for isotope monitoring of nuclear materials, forensics, geochemistry, planetary exploration and in the pharmaceutical industry. Laser-induced breakdown spectroscopy is the traditional technique for standoff analysis of solid materials [1], which either offers a broadband measurement with moderate spectral resolution or a narrow bandwidth measurement with high spectral resolution. Emission spectroscopy also relies on the population in the excited level, and the signal strength decreases as plasmas cool down. Moreover, emission spectroscopy suffers from self-absorption, which broadens the linewidth for optically thick plasmas. Dual frequency comb spectroscopy (DCS) offers the advantage of broad spectral coverage with high spectral resolution. As an absorptive spectroscopy technique, it can detect transitions regardless of excited state populations, and the signal strength corresponds directly with the number densities in the beam path. We have recently shown the efficacy of this technique in optically probing laser-induced plasmas by detecting rubidium and potassium simultaneously in the plasma generated by ablating a solid sample [2]. DCS allows the transient events of laser-induced plasmas to be probed with high temporal resolution for better characterization of the time-evolution of multiple species present in the sample simultaneously. Also, characteristics of the plasma, such as number densities, temperature, and path length, can be calculated from the absorption data. Here, we present results in which we ablate various samples and simultaneously detect the spectral amplitude and phase of the probe beam.

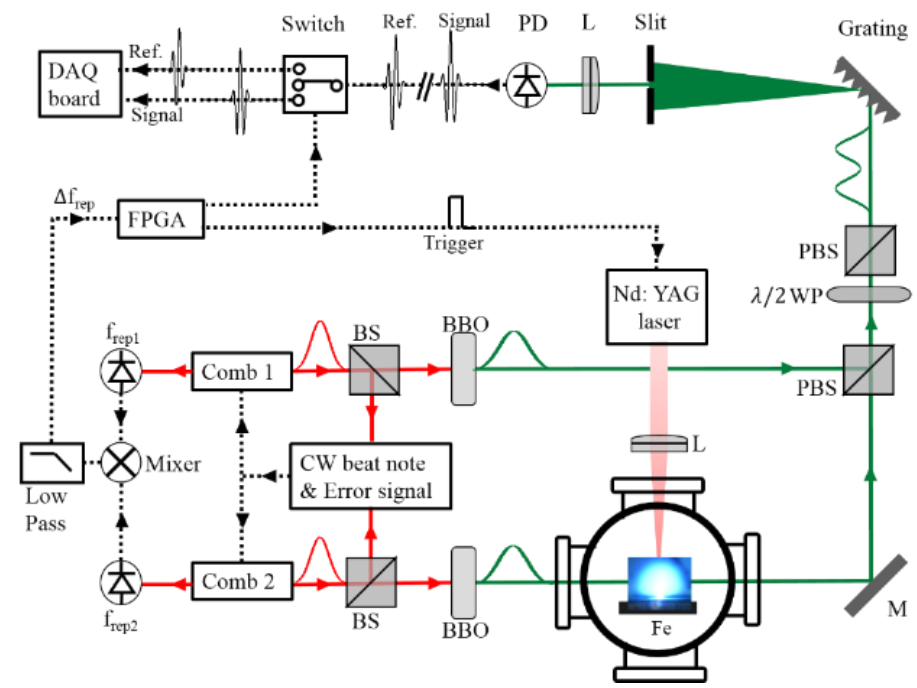

Figure 1. Schematic of the dual-comb laser system used to perform time-resolved phase spectroscopy of laser-induced plasmas. A pair of $\mathrm{Yb}$ fiber lasers generate frequency combs 1 and 2 which are both stabilized via optical phase-locking utilizing a $1064 \mathrm{~nm} \mathrm{CW}$ source. BBO crystals double the frequency of the combs, and the probe comb is sent through the ablation plasma and then interfered with the local oscillator comb. The two combs are spectrally filtered and then detected by a photodetector. The repetition rates of the two lasers are mixed to retrieve the difference frequency, which is sent to an FPGA to control the timing of the experiment components. The ablation laser is triggered to fire with a specific delay relative to one dual-comb interferogram, and electronic switches are used to select this and the previous interferogram detected by the photodetector. These are digitized, and the background and absorption spectra are calculated by Fourier transform. 


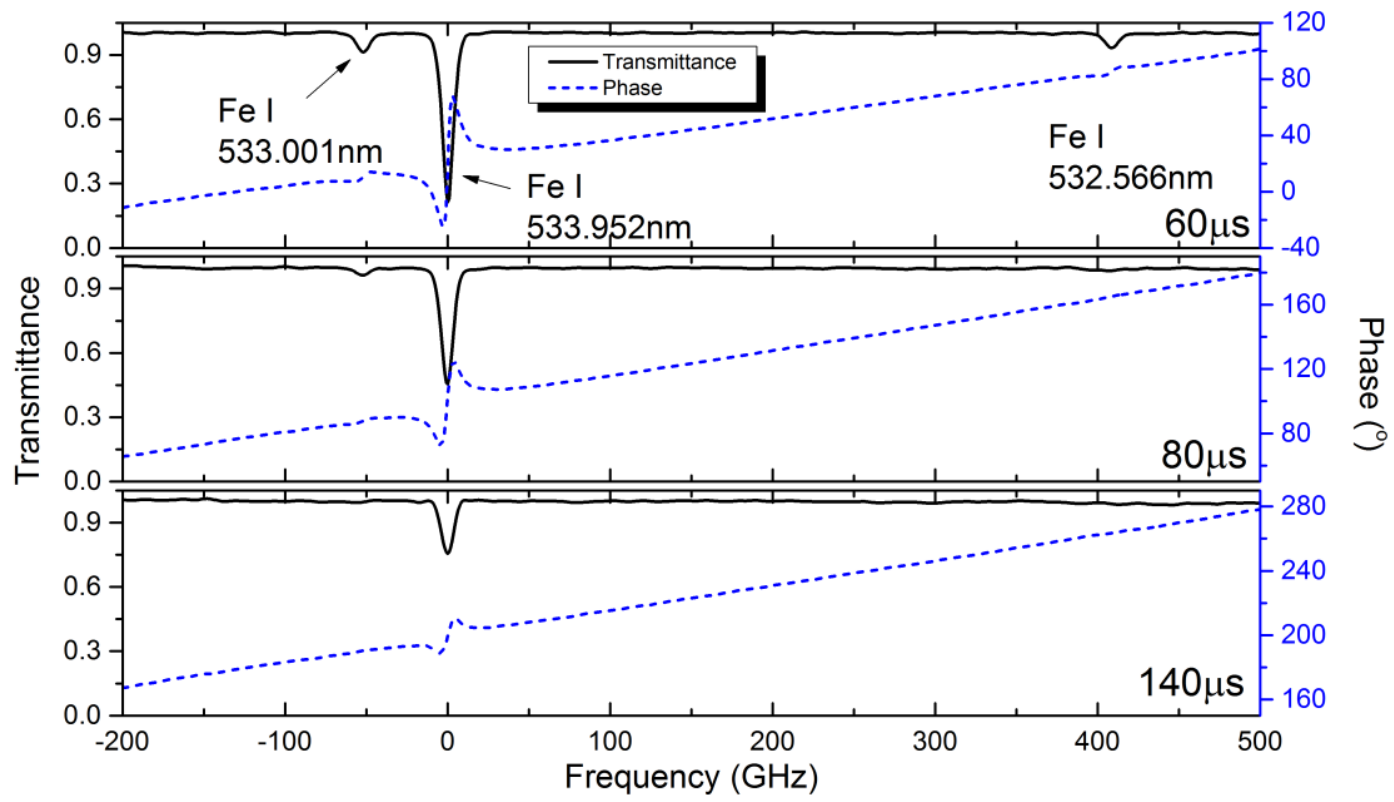

Figure 2. Plots showing the transmission and phase of the probe beam after passing through the ablated Fe plasma sample at three delay times. The frequency axis is plotted relative to the strongest Fe absorption transition.

The dual-comb laser system consists of two ytterbium fiber lasers with repetition rates of about $78 \mathrm{MHz}$ with a repetition rate difference $\Delta f_{\text {rep }}$ of about $500 \mathrm{~Hz}$. To study the iron transitions near $533 \mathrm{~nm}$, the $1064 \mathrm{~nm}$ centered lasers are frequency doubled using BBO crystals with $500 \mu \mathrm{m}$ thickness. We use an asymmetric configuration in which one comb is used to optically probe the plasma and is then interfered with the second comb, which serves as a local oscillator (Fig. 1). The plasma is generated within a vacuum chamber by ablating a stainless steel block under 100 Torr of flowing argon. The ablation laser is a Q-switched Nd:YAG operating at $10 \mathrm{~Hz}$ producing $\sim 9 \mathrm{~ns}, \sim 500 \mathrm{~mJ}$ pulses focused onto the target by a $12.5 \mathrm{~cm}$ lens. A cross-correlation signal is generated as one pulse samples the other, which is detected by a simple photodetector after spectral filtering. The interferograms are recorded over a time window of $20 \mu$ s with a 14-bit data acquisition card at a sample rate of $125 \mathrm{Ms} / \mathrm{sec}$. Taking the Fourier transform of the digitized interferogram gives the combined spectra of the two combs. Averaging multiple interferograms in the frequency domain increases the signal-to-noise ratio, and the interferograms just before each ablation event are recorded on a separate digitizer to calculate the background spectrum. The plasma is probed at different times after the ablation event by adjusting the electronic delay of the ablation laser's trigger signal. In our asymmetric configuration in which the absorption information is imprinted on only one comb, the phase information can be extracted from the interference of the two combs.

The time-resolved transmission spectra of three Fe I transitions and their corresponding phase detected following laser ablation of a stainless steel sample are shown in Figure 2. The transmission increases as the populations in these excited states decrease and the atoms travel outward out of the beam path. In this type of measurement, the phase information aids in discriminating between weak absorption lines and noise. Also, with the use of a phase reference, the phase can be used to calculate the plasma density as related to its refractive index, assuming a uniform plasma along the beam path. Measuring the plasma density using absorption spectroscopy at various times is a step toward better understanding the mechanics of plasmas and the species concentrations present in a solid material.

Acknowledgements This material is based upon work supported by the Air Force Office of Scientific Research under award number FA9550-15-1-0091 and the Defense Threat Reduction Agency under Grant No. HDTRA11710030. The Pacific Northwest National Laboratory is operated for the U.S. Department of Energy (DOE) by the Battelle Memorial Institute under Contract No. DE-AC05-76RL01830.

\section{References}

[1] S. S. Harilal, et. al., "Optical Spectroscopy of laser-produced plasmas for standoff isotopic analysis," App. Phys. Rev. 5, 021301 (2018).

[2] J. Bergevin, et. al., "Dual comb spectroscopy of laser induced plasmas," Nat. Commun. 9, 1273 (2018). 